\title{
Expansion of the Spectrum of FLNA Mutations Associated with Melnick-Needles Syndrome
}

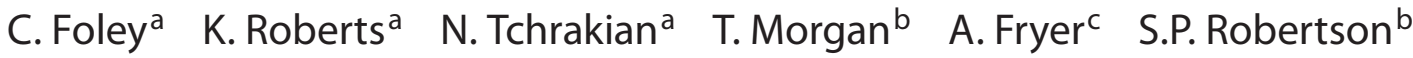 \\ N. Tubridy ${ }^{a}$ \\ ${ }^{a}$ Department of Neurology, St. Vincent's University Hospital, Dublin, Ireland; ${ }^{b}$ Department of \\ Paediatrics and Child Health, Dunedin School of Medicine, University of Otago, Dunedin, New Zealand; \\ 'Department of Clinical Genetics, Royal Liverpool Children's Hospital, Liverpool, UK
}

\section{Key Words}

Filamin A - Melnick-Needles syndrome - Multiple sclerosis • Stroke

\begin{abstract}
Melnick-Needles syndrome (MNS) is a rare X-linked bone dysplasia characterised by facial dysmorphology and radiographic abnormalities [Melnick and Needles, 1966;97:3948]. Previously, all published cases of MNS were associated with only 4 mutations [Robertson et al., 2003;33:487-491; Santos et al., 2010;152A:726-731], all localised within exon 22 of FLNA, the gene encoding the cytoskeletal protein filamin A. Here we report 3 new mutations in FLNA that are associated with MNS. One affected member of the first family with the mutation p.Y1229S presented with a stroke while this patient's daughter, previously known to be affected from a young age, developed multiple sclerosis. A second unrelated patient with a typical phenotype is shown to have the mutation c.1054G>T (p.G352W) within exon 7 of FLNA. A third individual with an atypical presentation but radiological findings very similar to those seen in classic MNS has a deletion likely to affect residues within repeat domain 14 . These findings indicate that the mutational spectrum for MNS is wider than previously appreciated and has implications for genetic testing strategies employed to confirm a diagnosis of this rare disorder.

Copyright $\odot 2010$ S. Karger AG, Basel
\end{abstract}

Melnick-Needles syndrome (MNS, OMIM 309350), one of the otopalatodigital syndrome spectrum disorders (OPSD) [Verloes et al., 2000], is an X-linked bone dysplasia. Patients are generally of short stature with craniofacial disproportion and normal intelligence [Robertson, 2004, 2005]. The facies are characterised by a prominent supraorbital ridge, exorbitism, oligohypodontia and micrognathia. Conductive deafness (due to ossicular malformation) and hydronephrosis are also commonly encountered. Radiological findings seen in MNS include late-closing fontanelles, skull base sclerosis, sinuous clavicles, irregular contour to the ribs, bowed long bones with cortical irregularity, angulation of the neck of the radius, tall vertebral bodies, pelvic hypoplasia with supra-acetabular constriction, and elongated metacarpals, metatarsals and phalanges. MNS is usually lethal in males in utero or in the perinatal period. In females, the clinical expression can be variable, even within families [Kristiansen et al., 2002].

MNS is caused by missense mutations in FLNA, the gene that encodes the actin-binding cytoskeletal protein filamin A [Robertson et al., 2003]. Filamin A associates with both the subcortical actin cytoskeleton and actin stress fibres within cells [Popowicz et al., 2006]. Filamin A has an $\mathrm{N}$-terminal actin-binding domain followed by a succession of 24 modular filamin repeats, interrupted at 2 sites (between domains 15 and 16 and also between do-

\section{KARGER}

Fax +4161306 1234 E-Mail karger@karger.ch www.karger.com
Niall Tubridy

Department of Neurology

St. Vincent's University Hospital

Elm Park, Dublin 4 (Ireland)

Tel. +353 1209 4817, Fax +353 1277 3506, E-Mail n.tubridy@svuh.ie 


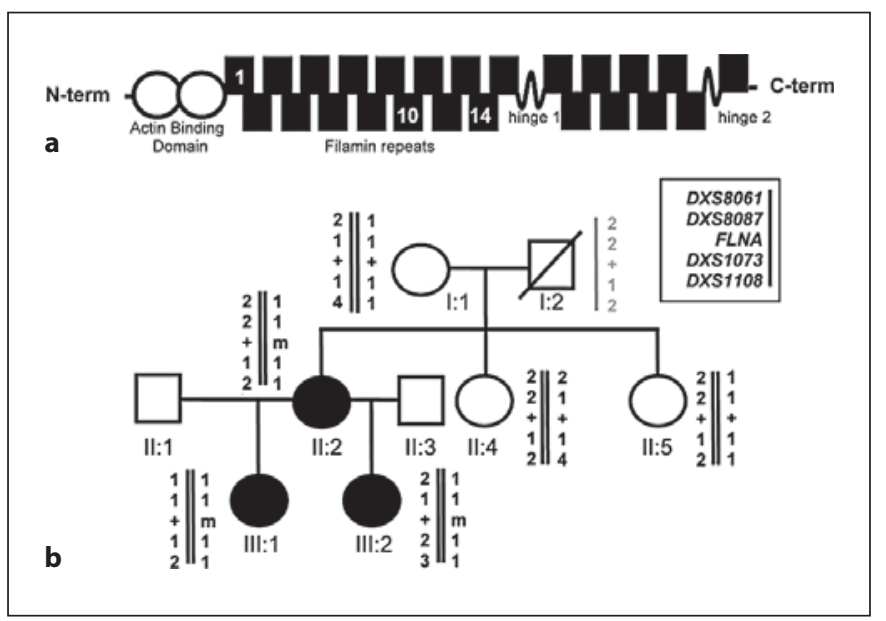

Fig. 1. a Schematic representation of the filamin A protein indicating the N-terminal actin-binding domain and the rod domain consisting of 24 filamin repeats interrupted by two flexible hinge regions. The location of repeats 1,10 and 14 in which the mutations are described in this report are indicated. $\mathbf{b}$ Pedigree of family including cases 1 and 2 . Genotyping with microsatellite markers demonstrated that the mutation c.3686A $>C(m)$ arose on a haplotype inherited from the unaffected individual I:1. The haplotype of individual I:2 in grey is inferred. Normal filamin A sequence, + .

mains 23 and 24) by flexible 'hinges' (fig. 1a). The mutations that underlie MNS all lead to substitutions in repeat 10 [Robertson et al., 2003]. Several lines of evidence suggest filamin participates in the remodeling of the cytoskeleton during development by integrating cell signaling events with subsequent alterations in cellular shape and motility [Popowicz et al., 2006]. MNS is allelic with 3 other skeletal dysplasias (otopalatodigital syndrome types 1 and 2 (OPD1, OPD2; OMIM 311300, OMIM 304120) and frontometaphyseal dysplasia (FMD, OMIM 305620)), and as a group they are collectively known as the otopalatodigital syndrome spectrum disorders. The mutations that cause these conditions are clustered within FLNA and are either missense or small deletions that preserve the translational reading frame. Such mutations lead to the production of full length filamin A protein that is expressed at levels equivalent to that seen in controls but confer a gain-of-function by increasing the affinity of filamin A for actin [Clark et al., 2009]. Phenotypes resulting from these mutations are readily discriminated from those associated with loss-of-function mutations in FLNA where the expression of FLNA protein is either diminished or absent [Robertson, 2005]. Females with truncating mutations exhibit a neuronal migration anomaly, bi- lateral periventricular nodular heterotopia $(\mathrm{PH})$ and demonstrate no skeletal anomalies [Fox et al., 1998]. Mutations that lead to complete loss of expression of FLNA in males confer embryonic lethality [Guerrini et al., 2004].

To date only 4 substitutions, G1176D, D1184E, A1188T, and S1199L, all clustered within exon 22 that encodes repeat 10 of the filamin A protein, have been shown to cause MNS [Robertson et al., 2003; Santos et al., 2010]. The G1176D and D1184E mutations have been described in single instances of the disorder with the other 2 mutations being highly recurrent. In this report we describe 3 unrelated families with MNS and previously unreported mutations remote from the exon 22 hotspot in FLNA. These data illustrate that the mutational spectrum underlying MNS is broader than previously appreciated and that molecular strategies to confirm a clinical diagnosis of MNS should take these observations into account.

\section{Methods}

The study was approved by the Otago Ethics Committee, Dunedin, New Zealand, and all patients or their guardians gave informed consent to participate. DNA was extracted from whole blood by standard procedures. Exon 2-48 of FLNA, including intron-exon junctions were amplified by PCR and subject to denaturing high performance liquid chromatography (DHPLC) according to the manufacturer's instructions (Transgenomik, Omaha, Nebr., USA). PCR products obtained from male subjects were mixed with products obtained from the same amplicon from a normal unrelated male control prior to DHPLC analysis. Samples demonstrating aberrant waveforms were subject to repeat amplification and sequenced using BigDye 3.1 chemistry and separated on an ABI3100 capillary sequencer. Microsatellite genotyping was performed using fluorescently labelled primers and products were separated on an ABI3100 sequencing and analysed using GeneMapper ver. 4.0 (Applied Biosystems).

\section{Clinical Cases}

Case 1 (II:2)

A 42-year-old right-handed woman (fig. 1) presented with a right-sided hemiparesis and global aphasia following a left middle cerebral artery stroke. She had been found at home conscious but unable to move or speak. The day before, she had complained of frontal headache, as well as nausea, vomiting and abdominal pain. There was no personal or family history of vascular disease or a clotting diathesis. Her only risk factor for stroke was a 
15-pack-per-year history of smoking. On admission she had a Glasgow Coma Score of 6/15, right-sided facial weakness, right-sided hemiparesis and an extensor right plantar response. Her blood pressure was $145 / 85 \mathrm{~mm} \mathrm{Hg}$. An extensive infarction in the territory of the middle cerebral artery was seen on a CT brain study. CT angiography and carotid ultrasound showed no evidence of an arterial dissection or significant plaque formation. Electrocardiography revealed normal sinus rhythm, and neither thrombus nor a patent foramen ovale were demonstrated on echocardiography. A thrombophilia screen was normal. A CT study of thorax, abdomen and pelvis was performed to exclude an underlying malignancy. There was marked cortic irregularity of the ribs, flaring of the ilia, mild scoliosis and bilateral minor modelling deformities of the lower femoral shafts (fig. 2). Her height is $152 \mathrm{~cm}$. There was also a moderate right-sided hydronephrosis (fig. 2). These features were suggestive of MNS given her daughter's subsequently revealed history (see case 2 below).

\section{Case 2 (III:1)}

The 23-year-old daughter of the index case (fig. 1) presented aged 6 years with a five-month history of severe sleep apnoea, weight loss and failure to thrive. She developed severe respiratory distress and required an emergency tracheostomy which remains in situ. Although her craniofacial abnormalities were considered the main causative factor of her respiratory symptoms at diagnosis mandibular advancement surgery was deemed unsuitable and a trial of CPAP was poorly tolerated. The patient had decreased hearing bilaterally and tinnitus. Her height was $144 \mathrm{~cm}$ and her facial features included a prominent supra-orbital ridge, exorbitism, mild hypertelorism, full cheeks, and micrognathia. At the age of 19 years, she presented with new onset weakness and incoordination of her right upper limb. Plain films of the abdomen revealed typical bony features of MNS (fig. 2). A magnetic resonance imaging (MRI) brain study demonstrated an enhancing lesion in the left centrum semiovale (fig. 2) and cerebrospinal fluid analysis revealed an elevated IgG index (0.960) with oligoclonal bands. Her symptoms responded to intravenous methylprednisolone. A subsequent MRI brain study performed 2 years later showed a left-sided periventricular lesion and some pericallosal lesions. She had no further neurological symptoms until aged 23 years when she reported a 3-4 week history of sensory disturbance in her right arm with concomitant loss of dexterity. Neurological examination performed shortly after

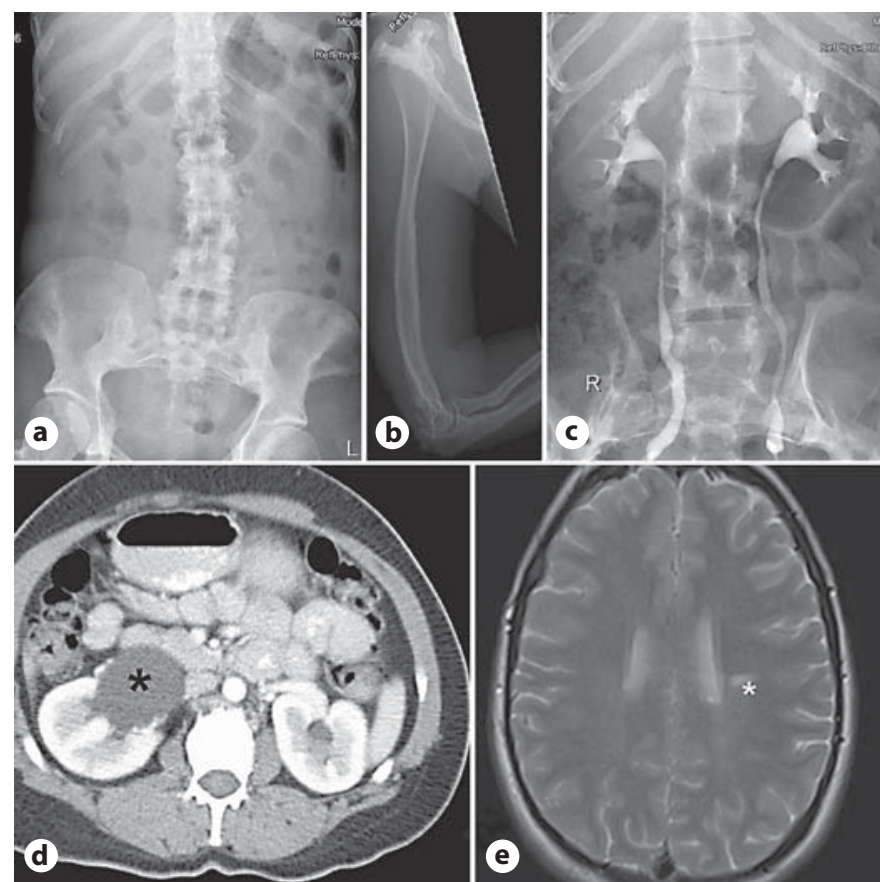

Fig. 2. Phenotype of cases 1 and 2. a Plain radiograph of II:2 showing ribbon-like ribs, mild scoliosis, flaring of the iliac wings and supra-acetabular constriction. b Cortical irregularity of the humerus and proximal ulna and radius in II:2. c Intravenous pyelogram on III:1 showing cortical irregularity of the ribs and flared iliac wings. d CT of the abdomen showing right-sided hydronephrosis $\left(^{*}\right)$ in II:2. e MRI scan of case 2 showing an enhancing lesion $\left({ }^{*}\right)$ consistent with the diagnosis of multiple sclerosis.

her symptoms had resolved was normal. A diagnosis of multiple sclerosis (MS) was confirmed. Examination of the second daughter of the proband (III:2) showed that she also had the typical clinical and radiological manifestations of MNS despite being asymptomatic (fig. 1). Analysis of exon 22 of FLNA demonstrated that case 1 was heterozygous for the mutation c.3686A $>C$ which predicts the substitution p.Y1229S. The substituted residue, Y1229, lies within filamin repeat domain 10 close to the 4 other confirmed mutations leading to this phenotype. It is conserved between filamin paralogues and across vertebrate orthologues. The mutation segregates with the radiological phenotype in this family. Examination of the segregation of microsatellite markers (DXS8061, DXS8087, DXS1073 and DXS1108) flanking the FLNA locus indicated that the mutation had arisen de novo on a haplotype that was inherited by II:2 from her unaffected mother I:1 (fig. 1). 


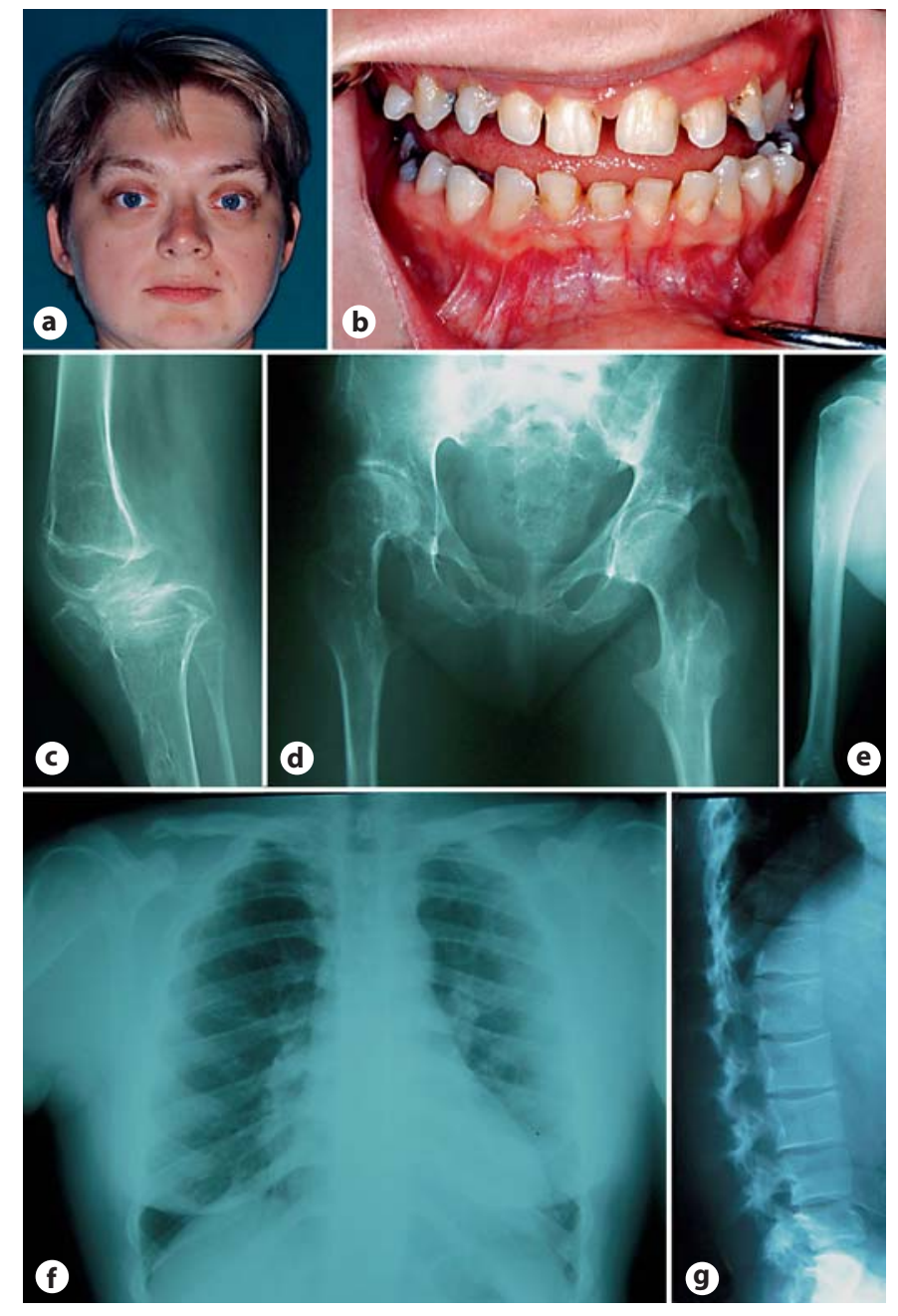

Fig. 3. Phenotype of case 3. a Supraorbital hyperostosis, round face, exorbitism and small chin. b Hypodontia with enamel hypoplasia. c Subluxed knee with cortical irregularity of the femur, tibia and patella. d Hypoplasia of the ischial and pubic rami, soft tissue calcification lateral to the femoral head and coxa valga. e Cortical irregularity of the upper humeral metaphysis. $f$ Narrow thorax with short broad clavicles. $g$ Increased height of the lumbar vertebral bodies except L5 which is dysplastic and irregular.

\section{Case 3}

This individual, the only person in her family to have MNS, has been previously described as a child in the literature [Dereymaeker et al., 1986] (case 3). She was born at term with a birth weight of $4.050 \mathrm{~kg}$ after a pregnancy complicated by polyhydramnios. Frequent respiratory infections dominated her early childhood. She developed pes cavus, progressive limitation of movement in the hips, knees and elbows and thoracic hypoplasia over the course of her childhood. She had mixed conductive and sensorineural hearing loss. Cognitive function was unimpaired. Now 32 years old she has limited mobility but maintains employment as an office manager. She develops frequent intercurrent respiratory infections although there is no stridor or fixed airway obstruction. Her teeth are small, brittle and have thin enamel. A CT scan demonstrated a bilateral deficiency of the modiolus and sclerosis of the incus and stapes as the basis for her hearing loss. Her height is $143 \mathrm{~cm}$. She has supraorbital hyperostosis, exorbitism, full eyebrows and a low anterior hairline (fig. 3). She has ulnar deviation of the wrists and spatulate thumbs. The chin is small. Her thorax is hypoplastic but there is no scoliosis. There is marked limitation of movement of both hips and limitation of flexion of the right knee. Her radiographs demonstrate skull base sclerosis, hypoplastic irregularly shaped clavicles, irregular contours to the ribs, irregular cortices of the long bones with mild bowing, subluxation of the right knee, hypoplastic ischial and pubic rami and flared iliac wings with supra-acetabular constrictions (fig. 3). The spine is straight but the lumbar vertebral bodies were increased in height although L5 is dysplastic and malformed (fig. 3). A de novo mutation, c.1054G $>$ T, was identified in exon 7 of FLNA, which predicts the substitution G352W in a central protein fold within repeat 1 of the filamin A protein.

\section{Case 4}

This female was the first born to unrelated parents. Her family history was unremarkable. She was first referred prior to surgery for craniosynostosis which took place at 4 months of age. The skull had an abnormal shape and a CT scan showed synostosis of the coronal, lambdoid and posterior sagittal sutures. There were fixed flexion deformities at the wrists and metacarpophalangeal joints and similar but milder deformities of elbows and knees. The forearms and forelegs were bowed. The genitalia were normal. Hip abduction was limited. Echocardiogram revealed an atrial septal defect. Ophthalmological examination showed corneal hypoplasia with a shallow anterior chamber, marked hypermetropia but normal fundi. A karyotype on blood leukocytes was 46,XX. A skeletal survey demonstrated a large anterior fontanelle, sinuous clavicles, cortical irregularity of the ribs, increased vertebral body height with an increased interpedicular distance. The iliac wings were flared with a supraacetabular constriction. The ischia were long and tapered. All long bones were bowed with flaring of the metaphyses (fig. 4). By 9 months of age she was developing a slight pectus excavatum. By 17 months her facial appearance was typical of MNS (fig. 4). A renal ultrasound examina- 
tion was performed at this age and was normal and her development was considered to be within the normal range although at 24 months she was only using single words. She died at 30 months of age from severe adult respiratory distress syndrome after surgery for hip dislocations. Analysis of FLNA demonstrated that she was heterozygous for the deletion c.4738_4755 + 10del28 spanning the exon 28 -intron 28 junction. No live cells were available to determine the consequences of deletion on splicing of the FLNA transcript. Exon 28 encodes for residues contributing to the $\mathrm{C}$-terminus of repeat 13 and the $\mathrm{N}$-terminus of repeat 14 of filamin A.

\section{Discussion}

The OPSD include OPD1, OPD2, FMD, and MNS [Robertson, 2005]. The conditions share much phenotypic overlap and are considered to encompass a spectrum. Severity ranges from mild manifestations in OPD1 to more severe presentations in FMD and OPD2. Prenatal lethality is most common in males with MNS and accordingly the female phenotype is typically the most severe of all the OPSD conditions although, as demonstrated in family 1 in this report, variable expressivity is a characteristic of the condition [Melnick and Needles, 1966; Kristiansen et al., 2002]. Before her stroke, the index case reported here had no medical history suggestive of MNS. Her daughter, however, was more severely affected. She had short stature and her facial and skeletal abnormalities were more pronounced. Her medical history was remarkable for failure to thrive, recurrent respiratory tract infections and obstructive sleep apnoea. Cases 1 and 2 are the first documented reports of premature stroke and MS respectively in association with MNS. Although loss-offunction mutations in FLNA are associated with stroke [Fox et al., 1998] and the mouse knock-out of this gene demonstrates vascular anomalies [Feng et al., 2006], there is little evidence for vascular defects in the gain-of-function OPSD conditions like MNS. A single instance of OPD2 in conjunction with a carotid artery-cavernous sinus fistula has been reported [Stratton and Bluestone, 1991] and some individuals with FMD have dysplastic cardiac valves [Robertson et al., 2006]. No such findings were observed in the individuals described here. The lack of an apparent association between stroke and MNS may be due to the small number of patients so far described with FLNA mutations as the basis for their disorder or may represent a chance occurrence. Similarly, susceptibility towards the development of MS may exist amongst

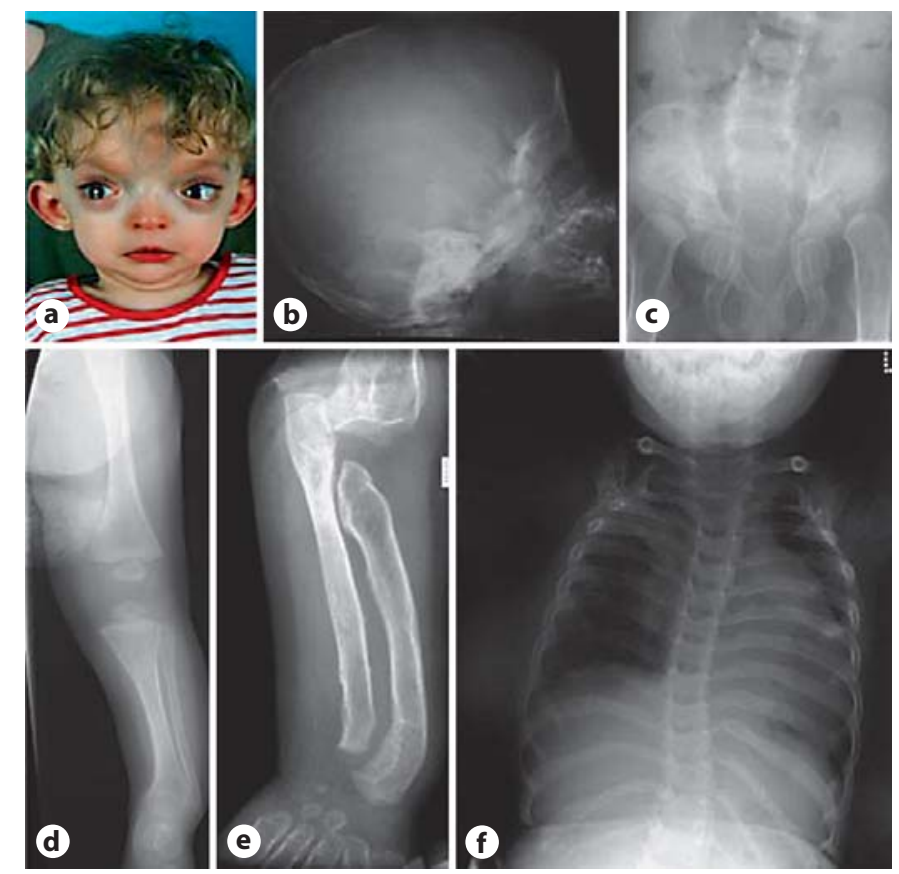

Fig. 4. Phenotype of case 4. a Broad bossed forehead, hypertelorism with exorbitism and micrognathia. b Skull base sclerosis and micrognathia. c Scoliosis, flared ilia with supra-acetabular constrictions, dysplastic ischial and pubic rami and coxa valga. d, e Bowing of the tibia and fibula in addition to pronounced dysplasia of the lower humerus, radius, ulna and metacarpals with absence of modelling and cortical sclerosis. $\mathbf{f}$ Scoliosis and irregular ribbon-like ribs.

individuals with FLNA mutation carriers, with filamin A known to interact with multiple regulators of immune function [Feng and Walsh, 2004]. Case 4 represents the first description of craniosynostosis in MNS, but further descriptions are required before this can be considered a confirmed association. The pathophysiology of the defects in ossification of the skull base and appendicular skeleton in the OPD spectrum disorders remains obscure.

Mutations that lead to the OPD spectrum disorders are substitutions or small deletions of amino acid residues that do not lead to down-regulation of expression of the filamin A protein [Clark et al., 2009]. These alterations occur in defined regions of the filamin A protein. Four mutations, all located within exon 22 and leading to substitutions in filamin repeat 10, lead to MNS. Two of them, A1188T and S1199L, are highly recurrent with the remaining 2 mutations occurring as single instances in the literature [Robertson et al., 2003; Santos et al., 2010]. This work further extends the mutational spectrum of 
the condition and indicates that molecular diagnosticians should extend their search for mutations that cause this condition to outside exon 22. Recently, the pathogenic mechanism underlying the very similar disorder OPD2 has been shown to lie in the enhancement of actin binding conferred by pathogenic mutations that result in substitutions within the actin binding domain of FLNA [Clark et al., 2009]. Additionally, data also now points to the role of filamin repeats in addition to the actin-binding domain in mediating actin binding by filamin [Nakamura et al., 2007]. Since OPD2 and MNS demonstrate considerable phenotypic similarity it seems possible that substitutions within repeat 10 (encoded by exon 22) and also sequences within repeat 1 and domains near hinge 1 that are likely to be affected by the mutations presented here, may also influence the binding of actin by filamin or the downstream consequences of enhancement of this interaction.

\section{References}

-Clark AR, Sawyer GM, Robertson SP, Sutherland-Smith AJ: Skeletal dysplasias due to filamin A mutations result from a gain-offunction mechanism distinct from allelic neurological disorders. Hum Mol Genet 18: 4791-4800 (2009).

Dereymaeker AM, Christens J, Eeckels R, Heremans G, Fryns JP: Melnick-Needles syndrome (osteodysplasty). Clinical and radiological heterogeneity. Helv Paediatr Acta 41: 339-351 (1986).

Feng Y, Walsh CA: The many faces of filamin: a versatile molecular scaffold for cell motility and signalling. Nat Cell Biol 6:1034-1038 (2004).

Feng Y, Chen MH, Moskowitz IP, Mendonza AM, Vidali L, et al: Filamin A (FLNA) is required for cell-cell contact in vascular development and cardiac morphogenesis. Proc Natl Acad Sci USA 103:19836-19841 (2006).

-Fox JW, Lamperti ED, Eksioglu YZ, Hong SE, Feng Y, et al: Mutations in filamin 1 prevent migration of cerebral cortical neurons in human periventricular heterotopia. Neuron 21: 1315-1325 (1998).
Guerrini R, Mei D, Sisodiya S, Sicca F, Harding $B$, et al: Germline and mosaic mutations of FLN1 in men with periventricular heterotopia. Neurology 63:51-56 (2004).

Kristiansen M, Knudsen GP, Soyland A, Westvik J, Orstavik KH: Phenotypic variation in Melnick-Needles syndrome is not reflected in X inactivation patterns from blood or buccal smear. Am J Med Genet 108:120-127 (2002).

Melnick JC, Needles CF: An undiagnosed bone dysplasia. A 2 family study of 4 generations and 3 generations. Am J Roentgenol Radium Ther Nucl Med 97:39-48 (1966).

- Nakamura F, Osborn TM, Hartemink CA, Hartwig JH, Stossel TP: Structural basis of filamin A functions. J Cell Biol 179:10111025 (2007).

Popowicz GM, Schleicher M, Noegel AA, Holak TA: Filamins: promiscuous organizers of the cytoskeleton. Trends Biochem Sci 31:411419 (2006).

Robertson SP: Molecular pathology of filamin A: diverse phenotypes, many functions. Clin Dysmorphol 13:123-131 (2004).

Robertson SP: Filamin A: phenotypic diversity. Curr Opin Genet Dev 15:301-307 (2005).
Robertson SP, Twigg SR, Sutherland-Smith AJ, Biancalana V, Gorlin RJ, et al: Localized mutations in the gene encoding the cytoskeletal protein filamin A cause diverse malformations in humans. Nat Genet 33:487-491 (2003).

Robertson SP, Jenkins ZA, Morgan T, Ades L, Aftimos S, et al: Frontometaphyseal dysplasia: mutations in FLNA and phenotypic diversity. Am J Med Genet A 140:1726-1736 (2006).

-Santos HH, Garcia PP, Pereira L, Leao LL, Aguiar RA, et al: Mutational analysis of two boys with the severe perinatally lethal MelnickNeedles syndrome. Am J Med Genet A 152A:726-731 (2010).

Stratton RF, Bluestone DL: Oto-palatal-digital syndrome type II with X-linked cerebellar hypoplasia/hydrocephalus. Am J Med Genet 41:169-172 (1991).

-Verloes A, Lesenfants S, Barr M, Grange DK, Journel H, et al: Fronto-otopalatodigital osteodysplasia: clinical evidence for a single entity encompassing Melnick-Needles syndrome, otopalatodigital syndrome types 1 and 2, and frontometaphyseal dysplasia. Am J Med Genet 90:407-422 (2000) 